\title{
Characterization and Antibiotic Sensitivity Pattern of Nonfermenting Gram Negative Bacilli from various Clinical Samples at a Tertiary Care Hospital
}

\author{
Narayan Shrihari and M. Iswarya* \\ Department of Microbiology, Vijayanagar Institute of Medical Sciences (VIMS), \\ Cantonment area, Ballari-583104, Karnataka, India
}

*Corresponding author

\section{Keywords \\ Non-Fermenting \\ Gram negative \\ Bacilli, Antibiotic \\ sensitivity, \\ Pseudomonas \\ aeurginosa, \\ Polymyxin B, \\ Meropenem.}

Article Info

Accepted:

15 August 2019

Available Online:

10 September 2019
A B S T R A C T

Non-fermenting Gram negative bacilli (NFGNB) are a group of bacteria which are aerobic, non sporing and most commonly they are saprophytes. They are also found as commensals in both man and animals. NFGNB are previously considered as a contaminant but now it is emerged as a important cause for life threatening nosocomial infections and also multidrug resistant organisms. Materials and methods: One year prospective study was conducted and our aim is to characterise the non fermenters from various clinical samples and their antibiogram. 110 isolates from various clinical samples with different age groups were included. The clinical samples like pus, urine, endotracheal aspirates, blood, sputum and body fluids were collected under aseptic precaution and identified by using standard protocol. It includes Gram's staining, Motility Testing, Catalase test, Oxidase test, OF test and various biochemical reactions. The susceptibility testing was done by Kirby bauer disc diffusion method. A total of 110 NFGNB were isolated and among that nonfermenters, Pseudomonas aeruginosa (49\%) was the predominant, followed by Acinetobacter baumanii (35\%), Acinetobacter lwoffi (7.3\%), Stenotrophomonas maltophilia (5.4\%), and Burkholderia cepacia (2.8\%). Pseudomonas aeruginosa showed good sensitivity to Polymyxin B (100\%), Meropenem and Imipenam (79.6\%) followed by Piperacillin tazobactam (72\%) and Amikacin (59.3\%). Acinetobacter baumanii showed 92\% sensitivity to Polymyxin B followed by $69 \%$ sensitivity to Meropenem and Imipenam. P.aeruginosa and A.baumanii were the commonest nonfermenters that are isolated in our study. They are associated with various infections like urinary tract infection, blood stream infections, surgical site infections and ventilator associated pneumonia. P.aeruginosa showed better sensitivity to following antibiotics: Polymyxin B, Meropenem, Imipenem and Amikacin. Therefore it is essential to identify the nonfermenters and to know their antibiotic sensitivity pattern.

\section{Introduction}

Non fermenting Gram Negative Bacilli (NFGNB) are a group of organisms which are aerobic and non-sporing that either do not use carbohydrates as a source of energy or degrade them through metabolic pathways other than fermentation. ${ }^{(1)}$

Most commonly these bacteria occur as 
saprophytes in the environment and also found as commensals in the human gut. ${ }^{(2)}$ These are ubiquitous in nature particularly in soil and water. Although frequently considered as contaminants, most of them have emerged as important nosocomial pathogens causing opportunistic infections in immunocompromised hosts. NFGNB accounts for about $15 \%$ of all bacterial isolates from a clinical microbiology laboratory. ${ }^{(3,4)}$

Non fermenting Gram Negative Bacilli can cause various infections including wound infections, urinary tract infections, meningitis, pneumonia, septicaemia, osteomyelitis, etc., Associated with risk factors like immunosuppression, neutropenia, mechanical ventilation, cystic fibrosis, indwelling catheters, invasive diagnostics and therapeutic procedures. Outcome of the disease mainly depends on prolonged hospital stay, broad spectrum antibiotic use and underlying host factors. $(5,6,8)$

In the National Nosocomial Infection Surveillance (NNIS) survey from the Centre for Disease Control and Prevention (CDC), Infections caused by non fermenters is the fourth most common cause of hospital acquired infections. ${ }^{(7)}$

This group of non fermenters includes Pseudomonas, Acinetobacter, Stenotrophomonas, Burkholderia, Alcaligenes and Weeksella spp, etc. Among these non fermenters, Pseudomonas aeruginosa is the commonly isolated non fermenter followed by Acinetobacter baumanii. Infection caused by these two nonfermenters is pathogenic for humans whereas infections caused by other species are less frequent. ${ }^{(3)}$

NFGNB show resistance to antibiotics due to production of extended spectrum $\beta$ lactamases and metallo $\beta$ lactamases. $(4,9, \quad 10)$ Nonfermenters can cause opportunistic as well as nosocomial infections and this nosocomial infections caused by nonfermenters are most commonly observed in debilitated and immune compromised patients. $(1,5,10)$

This study was undertaken to identify the nonfermenters isolated from various clinical samples and their antimicrobial susceptibility pattern.

\section{Materials and Methods}

The present study was undertaken at the Department of Microbiology, Vijayanagara Institute of Medical Sciences, Ballari for a period of one year. A total of 110 non fermenters were isolated from various clinical samples such as pus, sputum, urine, blood, ET tube and body fluids were included.

Identification is mainly based on the Gram staining, Motility testing, and growth on Nutrient Agar, Mac Conkey Agar and Blood Agar. The isolates which are catalase positive, oxidase positive or negative, non lactose fermenting colonies on Mac Conkey agar were identified by colony morphology and pigment production. They were inoculated in Triple sugar iron (TSI) agar slope. The colonies which failed to acidify the TSI agar were considered as non fermenters and subjected to the following tests such as Indole, Citrate, Urease, Nitrate reduction, growth at $42{ }^{\circ} \mathrm{C}$ and sensitivity to Polymyxin B. ${ }^{(1)}$

The sensitivity test was performed by Kirbybauer disc diffusion method using commercially available discs (Himedia). The results were interpreted as per the CLSI guidelines. (11) Pseudomonas aeruginosa ATCC 27853 was used as control strain.

\section{Results and Discussion}

A total of 110 non fermenters were isolated from various clinical samples. Among the 110 
non fermenters, $43(39 \%)$ were isolated from pus, $20(18.1 \%)$ were from urine, $19(17.2 \%)$ from wound swab, 11 (10\%) from blood, 9 $(8.1 \%)$ from sputum, 5 (4.5\%) from endotracheal aspirate and $3(2.7 \%)$ from body fluids. (Table: 1)

Majority of isolates of non fermenters were from Surgical ward $(40 \%)$ followed by ICU (20\%), Medicine (14.6\%), OBG (10\%), Urology (8\%), Burns (5.5\%), Ortho (4.6\%), Paediatrics (3.6\%), Otorhinolaryngology (2.7\%), TB ward (0.9\%), Dermatology (0.9\%). (Table: 2$)$

Among the nonfermenters, Pseudomonas aeruginosa (49\%) was the predominant isolate followed by Acinetobacter baumanii (35\%), Acinetobacter lwoffi (7.3\%), Stenotrophomonas maltophilia (5.4\%), and Burkholderia cepecia (2.8\%). (Table: 3 )

Pseudomonas aeruginosa showed good sensitivity to Polymyxin B (100\%), Meropenem and Imipenam (79.6\%) followed by Piperacillin tazobactam (72\%) and Amikacin (59.3\%). Acinetobacter baumanii showed $92 \%$ sensitivity to Polymyxin B followed by $69 \%$ sensitivity to Meropenem and Imipenam.

Among all the isolates maximum resistance was recorded for Gentamycin (61.8\%), Cotrimoxazole (60\%), followed by Ciprofloxacin $(50.9 \%)$ and Cefotaxime (47.3\%). (Table: 4)

Non fermenting Gram Negative bacilli (NFGNB) are being isolated with increasing frequency from various clinical samples. In recent years, the failure to treatment due to their multidrug resistance has led to the interest to carry out this study. In the present study out of 110 non fermenters, 43(39\%) were isolated from pus, 20 (18.1\%) from urine, $19(17.2 \%)$ wound swab, 11(10\%) blood, 9(8.1\%) sputum, $5(4.5 \%)$ from endotracheal aspirate and 3(2.7\%) from body fluids. In a study conducted by Gokale etal they reported that $58.4 \%$ non fermenters were isolated from pus/wound discharge followed by $23 \%$ from blood, $8.2 \%$ from urine, $4.5 \%$ from sputum and $2.3 \%$ from pleural fluid. ${ }^{(2)}$

A study conducted by Kirtilaxmi etal also stated that the isolation rate of pus was $21 \%$, $11 \%$ from urine, $7 \%$ from blood and $17 \%$ from tracheal aspirate. ${ }^{(3)}$

In another study conducted by Kalidas etal they observed that the isolation rate was $27.9 \%$ from pus sample, $18.4 \%$ from tracheal aspirate, $16.4 \%$ from sputum and $16.4 \%$ from blood and $15.9 \%$ from urine. ${ }^{(12)}$

In the present study maximum number of non fermenters were isolated from Surgical wards $(28.2 \%)$ followed by Intensive care unit $(20 \%)$ and Medicine ward (14.6\%). Similar findings were also reported by Anupurba et al., that higher prevalence rate of non fermenters was observed in surgery wards $(29.9 \%)$.

The second highest prevalence observed in this study was intensive care units (20\%) followed by Medical ward (14.6\%).

In another study conducted by Keertilaxmi B etal they also reported that the isolation of NFGNB from intensive care units was $37 \%$. (3) Among the non fermenters in recent years there are outbreaks of Burkholderia cepacia complex septicaemia have been documented worldwide in intensive care units (ICUs), oncology units and renal failure patients. ${ }^{(13)}$

In this present study, the commonest NFGNB isolated were Pseudomonas aeruginosa 54(49\%) followed by Acinetobacter baumanii 39(35\%), Acinetobacter lwoffi 8(7.3\%), S.maltophilia 6 (5.4\%), and Burkholderia cepecia 3(2.8\%). 
Table.1 Nonfermenting gram negative bacilli from various clinical samples

\begin{tabular}{|c|c|c|}
\hline Clinical Samples & No. of Isolates & $\%$ of Isolates \\
\hline Pus & 43 & $39 \%$ \\
\hline Urine & 20 & $18 \%$ \\
\hline Wound swab & 19 & $17 \%$ \\
\hline Blood & 11 & $10 \%$ \\
\hline Sputum & 9 & $8.5 \%$ \\
\hline ET tube & 5 & $4.6 \%$ \\
\hline Body fluids & 3 & $2.8 \%$ \\
\hline Total & 110 & $100 \%$ \\
\hline
\end{tabular}

$(n=110)$

Table.2 Distribution of clinical isolates

\begin{tabular}{|c|c|c|}
\hline Speciality & Clinical Isolates & Percentage (\%) \\
\hline Surgery & 32 & 29.1 \\
\hline Intensive care unit & 22 & 20 \\
\hline Medicine & 16 & 14.6 \\
\hline OBG & 11 & 10 \\
\hline Urology & 9 & 8.2 \\
\hline Burns & 6 & 5.4 \\
\hline Ortho & 5 & 4.6 \\
\hline Paediatrics & 4 & 3.6 \\
\hline Otorhinolaryngology & 3 & 2.7 \\
\hline TB ward & 1 & 0.9 \\
\hline Dermatology & 1 & 0.9 \\
\hline Total & 110 & 100 \\
\hline
\end{tabular}

$(\mathrm{n}=110)$

Table.3 Speciation of nonfermenting gram negative bacilli

$(n=110)$

\begin{tabular}{|l|c|c|}
\hline \multicolumn{1}{|c|}{ Organisms } & No. of Isolates & Percentage (\%) \\
\hline Pseudomonas aeurginosa & 54 & 49 \\
\hline Acinetobacter baumanii & 39 & 35 \\
\hline Acinetobacter lwoffi & 8 & 7.3 \\
\hline $\begin{array}{l}\text { Stenotrophomonas } \\
\text { maltophilia }\end{array}$ & 6 & 5.4 \\
\hline Burkholderia cepacia & 3 & 2.8 \\
\hline \multicolumn{1}{|c|}{ Total } & 110 & 100 \\
\hline
\end{tabular}


Table.4 Antimicrobial susceptibility pattern of nonfermenting gram negative bacilli

\begin{tabular}{|c|c|c|c|c|c|c|c|c|c|c|}
\hline \multirow[t]{2}{*}{ Antibiotics } & \multicolumn{2}{|c|}{$\begin{array}{c}\text { P.aeruginosa } \\
(\mathrm{n}=54)\end{array}$} & \multicolumn{2}{|c|}{$\begin{array}{c}\text { B.cepecia } \\
(n=3)\end{array}$} & \multicolumn{2}{|c|}{$\begin{array}{c}\text { A.baumanii } \\
(n=39)\end{array}$} & \multicolumn{2}{|c|}{$\begin{array}{c}\text { A.lwoffi } \\
(\mathrm{n}=8)\end{array}$} & \multicolumn{2}{|c|}{$\begin{array}{c}\text { S.maltophilia } \\
(\mathrm{n}=6)\end{array}$} \\
\hline & $\mathrm{S}$ & $\%$ & $\mathrm{~S}$ & $\%$ & $\mathrm{~S}$ & $\%$ & $\mathrm{~S}$ & $\%$ & $\mathrm{~S}$ & $\%$ \\
\hline Gentamicin & 22 & 40.7 & - & - & 18 & 46 & 4 & 50 & - & - \\
\hline Amikacin & 32 & 59.3 & - & - & 26 & 66 & 6 & 80 & 2 & 33.3 \\
\hline Ciprofloxacin & 23 & 42.6 & 1 & 33.3 & 18 & 46 & 4 & 50 & 6 & 100 \\
\hline Ofloxacin & 23 & 42.6 & 1 & 33.3 & 18 & 46 & 4 & 50 & 6 & 100 \\
\hline Ceftazidime & 30 & 56 & 1 & 33.3 & 20 & 51 & 6 & 80 & - & - \\
\hline Cefotaxime & - & - & 1 & 33.3 & 20 & 51 & 6 & 80 & - & - \\
\hline $\begin{array}{l}\text { Piperacillin- } \\
\text { tazobactam }\end{array}$ & 39 & 72 & 1 & 33.3 & 26 & 66 & 8 & 100 & 1 & 16.7 \\
\hline Cotrimoxazole & - & - & 3 & 100 & 20 & 51 & 4 & 50 & 6 & 100 \\
\hline Imipenam & 43 & 79.6 & 2 & 66.7 & 27 & 69 & 8 & 100 & - & - \\
\hline Meropenem & 43 & 79.6 & 2 & 66.7 & 27 & 69 & 8 & 100 & - & - \\
\hline Polymyxin B & 54 & 100 & - & - & 36 & 92 & 8 & 100 & 6 & 100 \\
\hline
\end{tabular}

*S-Sensitive and *- Not tested

A study conducted by Kalidas et al., also reported that Pseudomonas aeruginosa $(50.2 \%)$ was the predominant isolate followed by A.baumannii (24.9\%), A.lwoffi (5.5\%), S.maltophilia (3\%) and Burkholderia cepacia (7\%). ${ }^{(12)}$ In the present study Pseudomonas spp and Acinetobacter spp were the commonest NFGNB isolated which correlates with other studies. ${ }^{(3,16,17)}$

Because of the prevalence of high intrinsic resistance of different NFGNB to different antimicrobial agents in recent years, the absolute identification of non fermenters and their resistance pattern should be performed at microbiology laboratory. As this can be an important guide for the clinicians for appropriate selection of empiric therapy. In this study, the antimicrobial susceptibility pattern of P.aeruginosa showed 54 (100\%) sensitivity to Polymyxin B, 43 (79.6\%) sensitivity to Imipenem and Meropenem followed by Piperacillin tazobactum 39 (72\%), Amikacin 32(59.3\%), Ceftazidime 30 (56\%), Ciprofloxacin and Ofloxacin 23(42.6\%) and Gentamycin 22 (40.7\%) which correlates with the study conducted by Grewal et al., ${ }^{(18)}$ and Kaur et al., ${ }^{(19)}$.

A Study from Gokale et al., also reported that most of the isolates of Pseudomonas aeruginosa were sensitive to Meropenem (96.2\%), followed by Ciprofloxacin (50.4\%) and Amikacin (49.5\%). ${ }^{(2)}$

A study conducted by Nautiyal etal reported that all the isolated Pseudomonas aeruginosa were sensitive to Polymyxin $B{ }^{(14)}$ which correlates with our study as well. Kirtilaxmi etal reported the sensitivity of Pseudomonas aeruginosa were ceftazidime (60\%), Gentamycin (65\%), Piperacillin-tazobactum (73.3\%), Imipenem (80\%), Amikacin (83.3\%), Ciprofloxacin $(58.3 \%){ }^{(3)}$ It is similar to our study.

In the present study, the isolates of A.baumannii showed 27 (69\%) sensitivity to Meropenem and Imipenem followed by Amikacin and Piperacillin tazobactum 26 (66\%), Ceftazidime and Cotrimoxazole 20 (51\%), Gentamycin and Ciprofloxacin and 
Ofloxacin $18(46 \%)$ each respectively. Most of the isolates were sensitive to Polymyxin B $36(92 \%)$. These results are similar to the study conducted by Nautiyal et al., ${ }^{(14)}$

In the present study, all the isolates of A.lwoffi were sensitive to Imipenem, Meropenem and Piperacillin tazobactum $8(100 \%)$ followed by Cefotaxime, Ceftazidime and Amikacin 6 (80\%), Gentamycin, Cotrimoxazole and Ciprofloxacin and Ofloxacin 4 (50\%), Polymyxin B (100\%). Similar to the present study, another study conducted by Nautiyal etal reported that all the isolated A.lwoffi were $100 \%$ sensitive to Polymyxin B. ${ }^{(14)}$

In our study, among the isolated S.maltophilia majority were sensitive to Cotrimoxazole, Ciprofloxacin and Ofloxacin and Polymyxin B 6 (100\%), followed by Amikacin 2(33.3\%) and Piperacillin tazobactum1 (16.7\%). These results are similar to another study conducted by Nautiyal etal They reported that all the isolated S.maltophilia were $100 \%$ sensitive to polymyxin B. (14) Similar results were also shown in another study conducted by Deepak etal They reported that all the isolated S.maltophilia showed $100 \%$ sensitivity to Ciprofloxacin and Cotrimoxazole and 33.3\% to piperacillin tazobactum and $16.67 \%$ to Gentamycin ${ }^{(15)}$ S. maltophilia is intrinsically resistant to most $\beta$ lactams, including carbapenems. ${ }^{(13)}$ In our study, among the isolates of Burkholderia cepacia (3), all were sensitive to Cotrimoxazole 3(100\%), followed by Imipenem and Meropenem 2(66.7\%), Ciprofloxacin, Ofloxacin, Cefotaxime and Ceftazidime 1(33.3\%). In contrast to the present study, Kalidas etal showed the $92.8 \%$ sensitivity to Imipenem and Cotrimoxazole, $85 \%$ to Ceftazidime and Ciprofloxacin and $57 \%$ to Piperacillin tazobactum. ${ }^{(12)}$

Burkholderia cepacia show intrinsic resistance to aminoglycosides and polymyxins due to the presence of inducible chromosomal $\beta$ - lactamases and altered penicillin-binding proteins leads to resistance to $\beta$-lactams.

They also mediate resistance to chloramphenicol, trimethoprim and fluoroquinolones through its antibiotic efflux pumps. ${ }^{(13)}$

Observations from the present study showed that aerobic NFGNB which are usually considered as contaminants are now emerging as important nosocomial pathogens. Different antimicrobial susceptibility pattern and multidrug resistance by nonfermenters cause difficulty in treating the infections. ESBL and MBL production by these organisms lead to high morbidity and mortality and we have only option of treating them by potentially toxic drugs like Colistin and Polymyxin B. All the health care institutions should have their own antimicrobial policy, regular surveillance and infection control protocols to avoid high incidence of resistant non fermenters.

\section{References}

Koneman EW, Allen SD. Colour atlas and Text book of diagnostic Microbiology, 6th ed, Philadelphia: Lippincott-Williams\& Wilkins Publishers; 2006.

Gokhale S, Metgud SC. Characterization and antibiotic sensitivity pattern of non fermenting gram negative bacilli from various clinical samples in a tertiary care hospital, Belgaum. J Pharm Biomed Sci. 2012; 17(17):1-5.

Kirtilaxmi KB, Padmavathy M, Malini J, Naveneeth BV. Prevalence of nonfermenting Gram-negative bacilli and their in vitro susceptibility pattern at a tertiary care teaching hospital. J Sci Soc. 2014; 41(3):162-6.

Mehta M, Punia JN, Joshi RM. Antibiotic resistance in Pseudomonas aeruginosa strains isolated from various clinical specimens- a retrospective study. Indian J Med Microbiol. 2001; 19(4):232.

Nagoba BS, Deshmukh SR, Ulka G etal Invitro 
susceptibility of Pseudomonas aeruginosa to different antibiotics. Indian J Med Microbio.1997; 15:185-6.

Mishra B, Bhujwala RA, Shrinivas. Non fermenters in human infections. Indian $\mathbf{J}$ Med Res. 1986 Jun; 83:561-6.

Quinn JP. Clinical problems posed by multiresistant nonfermenting gramnegative pathogens. Clin Infect Dis. Suppl. 1998; 1: 117-24.

Bergogne Berezin E, Towner KJ. Acinetobacter spp. as Nosocomial Pathogens: Microbiological, Clinical and Epidemiological Features. Clin Microbiol Rev. 1996; 9(2):148-65.

Rubin SJ, Granato PA and Wasilaskas BL. Glucose nonfermenting gram negative bacteria. In: Lennette EH, Balwos A, Hausler Jr. WJ and Shadomy HJ, editors. Manual of clinical Microbiology. $4^{\text {th }}$ edition, Washington, DC: American society for Microbiology; 1985.

Gardner, B Griffin, Swartz, Kunz. Nonfermentative gram negative bacilli of nosocomial interest. American J Med. 1970 Jun; 48:735-49.

Clinical and Laboratory Standard Institute. Performance Standards for antimicrobial susceptibility testing, $28^{\text {th }}$ International Supplement (M100-S28). Wayne; Pennsylvania.USA: 2018.

Kalidas Rit, Falguni Nag, Hirak Jyoti Raj, PK Maity. Prevalence and Susceptibility Profiles of Non-fermentative Gram-negative Bacilli Infection in a Tertiary Care Hospital of Eastern India. Indian Journal of Clinical Practice.2013; 24:5.

. Gautam V, Ray P, Vandamme P, Sharma M etal Identification of lysine positive nonfermenting gram negative bacilli Stenotrophomonas maltophilia and Burkholderia cepacia complex. Indian J
Med Microbiol. 2009; 27(2): 128-30.

Nautiyal S, Jauhari S, Goel N, Mahawal BS. Current trend of Non fermenting Gram Negative Bacilli in a Tertiary Care Hospital in Dehradun, Uttarkhand. International Journal of Advanced Research. 2014; 2(2): 322-28.

Deepak Juyal, Rajat Prakash, Shamanth A, Shankarnarayan, Munesh Sharma, Vikrant Negi, Neelam Sharma. Prevalence of nonfermenting gram negative bacilli and their in vitro susceptibility pattern in a tertiary care hospital of Uttarkhand: A study from foothills of Himalayas. Saudi journal for Health Sciences. 2013; 2 (2):108-12.

Ruchita Mahajan, Neeraj, Sarika, Bella Mahajan. Isolation and Identification of Non Fermenting Gram Negative Bacilli in A Tertiary Care Hospital. Sch. J. App. Med. Sci. 2016; 4(3):872-76.

Gomathi Maniyan, Dillirani Vedachalam, Nithya Chinnusamy. Characterisation and antimicrobial susceptibility pattern of nonfermenting gramnegative bacilli from various clinical samples in a tertiary care hospital. Indian J Microbiol Res. 2016; 3(4):387-91.

Udhayir Singh Grewal, Rupinder Bakshi, Geeta Walia, Parth Rajeshbhai Shah. Antibiotic susceptibility profiles of nonfermenting gramnegative bacilli at a tertiary care hospital in Patiala, India. Niger Postgrad Med J. 2017; 24:121-25.

Amandeep kaur, Amarjit kaur gill, Satnam singh. Prevalence and antibiogram of nonfermenting gramnegative bacilli isolates obtained from various clinical samples in a tertiary care hospital, Bathinda, Punjab, India. Int J Res Med Sci. 2018; 6(4): 122834.

\section{How to cite this article:}

Narayan Shrihari and Iswarya M 2019. Characterization and Antibiotic Sensitivity Pattern of Nonfermenting Gram Negative Bacilli from various Clinical Samples at a Tertiary Care Hospital. Int.J.Curr.Microbiol.App.Sci. 8(09): 1502-1508.

doi: https://doi.org/10.20546/ijcmas.2019.809.172 